\title{
Comparing the diagnostic efficacy of digital breast tomosynthesis with full-field digital mammography using BI-RADS scoring
}

\author{
Rana M. Naeim ${ }^{*}$ (D), Rania A. Marouf, Merhan A. Nasr and Marwa E. Abd El-Rahman
}

\begin{abstract}
Background: Mammography has been the mainstay for the detection of breast cancer over decades. It has gradually advanced from screen film to full-field digital mammography. Tomosynthesis has evolved as advanced imaging for early diagnosis of breast lesions with a promising role in both diagnostic and screening settings, particularly in dense and treated breasts.

Results: This study included 90 female patients according to our inclusion criteria. All patients perform full-field digital mammography (FFDM) and digital breast tomosynthesis (DBT) and were classified according to breast density and age groups. Breast imaging reporting and data system (BI-RADS) scoring was assigned for each case. This was correlated with the final diagnosis; the diagnostic indices of mammography were a sensitivity of $64.44 \%$, a specificity of $77.78 \%$, a positive predictive value (PPV) $74.63 \%$, a negative predictive value (NPV) of $68.63 \%$, and a diagnostic accuracy of $71.11 \%$. Diagnostic indices of DBT were a sensitivity of $100 \%$, a specificity of $97.77 \%$, PPV $97.78 \%$, NPV 100\%, and diagnostic accuracy of $97.7 \%$.

In patients with dense breasts American College of Radiology (ACR) (c and d), 61\% of cases had changed their BIRADS scoring with the addition of tomosynthesis. Yet, in non-dense breast ACR (a and b), 45\% of cases had changed BIRADS scoring with the addition of DBT to FFDM.

Conclusion: DBT is a promising imaging modality offering better detection and characterization of different breast abnormalities, especially in young females, and those with dense breasts with an increase of sensitivity and specificity than FFDM. This leads to a reduction in the recalled cases, negative biopsies, and assessing the efficacy of therapy as it enables improving detection of breast cancer and different breast lesions not visualized by conventional mammography
\end{abstract}

Keywords: Mammography, Tomosynthesis, Dense breast

\section{Background}

Breast cancer incidence had increased by $20 \%$ with a possible increase of diagnosis before the age of 50. The pursuit of correct and cost-effective ways to diagnose breast cancer early remains of interest [1]. Cancer care had been individualized for patients, and thus, better characterization was required for treatment planning.

\footnotetext{
* Correspondence: ranamamdouh89@gmail.com

Radio-diagnosis Department, Faculty of Medicine, Ain Shams University, Cairo, Egypt
}

(c) The Author(s). 2021 Open Access This article is licensed under a Creative Commons Attribution 4.0 International License, which permits use, sharing, adaptation, distribution and reproduction in any medium or format, as long as you give appropriate credit to the original author(s) and the source, provide a link to the Creative Commons licence, and indicate if changes were made. The images or other third party material in this article are included in the article's Creative Commons licence, unless indicated otherwise in a credit line to the material. If material is not included in the article's Creative Commons licence and your intended use is not permitted by statutory regulation or exceeds the permitted use, you will need to obtain permission directly from the copyright holder. To view a copy of this licence, visit http://creativecommons.org/licenses/by/4.0/.

Imaging examination is an important tool to help diagnose and decide therapeutic response [2].

Screening mammography is considered the primary technique, and the most important screening tool in breast cancer detection and assessment. It was responsible for a reduction in mortality among the age group of 40 years or older [3]. Initially, screen-film mammography was done, but today, the most common two-view examination (mediolateral oblique and craniocaudal) using full-field digital mammography (FFDM) used, 
searching for any mass, architectural distortion, or calcification, and then giving BIRADS score [4].

Nevertheless, mammography suffers from several limitations due to the low contrast between tumors and surrounding tissue [5]. Especially in dense breasts, this can lead to a decrease in sensitivity, and added imaging methods are necessary [6]. Advances in full-field digital mammography (FFDM) led to the development of digital breast tomosynthesis, which is a three-dimensional breast examination. The multi-view information from the multiple low-dose images used to generate thin slices (at 1-mm spacing) that viewed sequentially as a stack in orientation, e.g., craniocaudal, mediolateral oblique with the potential to improve accuracy by improving differentiation between malignant and non-malignant lesions [6].

The primary operational advantage of tomosynthesis is that the procedure is very similar to the conventional mammography examination in the technologist's tasks and the woman being imaged, yet it eliminates the limitation of full-field digital mammography by overlapping breast tissue. Therefore, tomosynthesis is implied easily in the current clinical practices with minor operational adjustments [7].

\section{Aim of the work}

Our study aims to evaluate the impact of digital breast tomosynthesis (DBT) in comparison to full-field digital mammography in improving the detection and characterization of different breast lesions and interpretations of BIRADS scoring in all breast densities at different age groups.

\section{Methods}

The study was a prospective study carried over 8 months with an extra 18 months when follow-up was needed in some cases. The study included 90 female patients with their ages ranged from 32 to 70 years (mean age $47.18 \pm$ 11.24 SD). Full-field digital mammography and digital breast tomosynthesis were followed by US examination done for all patients.

\section{Subjects}

Patients included in this study those referred from breast clinic for either:

- Screening purposes: whether primary screening or those who had already undergone treatment for breast cancer and were on yearly follow-up.

- Diagnostic purposes: women presenting with a palpable lump, or any other breast complaints such as nipple discharge or breast pain.

\section{Exclusion criteria:}

- Pregnant and lactating women

- Those having open breast wounds

\section{The technique of full-field digital mammography}

During acquisition, the breast was compressed between breastplates and standard views medio lateral-oblique and craniocaudal views were taken for all patients.

\section{The technique of 3D tomosynthesis}

During acquisition, the breast was compressed between breastplates as in conventional mammography, and the $\mathrm{X}$-ray tube pivoted in an arc that varies between $15^{\circ}$ (narrow range) and $60^{\circ}$ (wide range) in a plane aligned with the chest wall allowing for 11 to 15 low-dose projection images (2D) acquired for the tomosynthesis images. Images of the tomosynthesis were obtained in the same standard projections (craniocaudal and mediolateral oblique) as conventional screening mammography. Data from the low-dose projection 2D images used to reconstruct 1-mm-thick sections separated by $1-\mathrm{mm}$ space to form the 3D volume of the compressed breast in the form of a series of images through the entire breast. Images were assessed on a dedicated workstation using Seno Bright spectral Priština, GE healthcare full-field digital mammography device. This varied according to the thickness of the compressed breast.

\section{Image analysis and interpretation}

Two experienced readers independently viewed and interpreted FFDM, synthetic 2D, and DBT. Each breast was evaluated about the presence of lesions or not, site of the lesions, type (mass, architectural distortion, focal asymmetry), margin definition, and \pm calcifications. Finally, the BIRADS category of the lesions in the imaging modalities individually determined according to the BIRADS lexicon 2013 classification (Table 1), and all cases were also categorized by breast density (according to ACR guidelines edition 2013) and age group. The obtained data were correlated with ultrasound examination.

The final diagnosis was obtained by histopathological assessment for lesions with BIRADS IV or more and those having BIRADS III further correlated with the ultrasound data, and then followed up (3 follow-up studies every 6 months).

True positive and true negative were decided by further diagnostic work-up, which included other imaging studies by ultrasonography, histopathological examination, or follow-up. 
Table 1 BIRADS assessment categories according to BIRADS atlas 2013 category assessment

\begin{tabular}{ll}
\hline BIRADS 0 & Incomplete-need additional imaging evaluation and/or prior mammograms for comparison \\
BIRADS 1 & Negative \\
BIRADS 2 & Benign \\
BIRADS 3 & Probably benign \\
BIRADS 4 & Suspicious 4A: low suspicion for malignancy 4B: moderate suspicion for malignancy 4C: high suspicion for malignancy \\
BIRADS 5 & Highly suggestive of malignancy \\
BIRADS 6 & Known biopsy-proven malignancy \\
\hline
\end{tabular}

\section{Statistical analysis}

Data were coded and entered using the statistical package SPSS (Statistical Package for the Social Sciences) version 26 (IBM Corp., Armonk, NY, USA). Data were summarized using frequency (count) and relative frequency (percentage). Standard diagnostic indices including sensitivity, specificity, positive predictive value (PPV), negative predictive value (NPV), and diagnostic efficacy were calculated as described by (Galen, 1980). For comparing categorical data, chisquared $\left(c^{2}\right)$ test performed an exact test used instead when the expected frequency is less than five (Chan, 2003). $P$ value of less than 0.05 was considered as statistically significant.

\section{Results}

In the current study, patients were divided into four groups according to breast density (Fig. 1) and according to the age group (Fig. 2). The distribution of different breast densities among different age groups is shown in (Fig. 3). Out of the total 90 cases, 50 were diagnostic and 40 were screening cases.

As regard lesion detection, classification, and BIRADS category for each case, FFDM detected lesions in $48 / 90$ cases $(53.3 \%)$ from which $39 / 48$ cases were classified as with malignant lesions (30/48 cases were given BIRADS score IV, 4/48 cases were given
BIRADS score $\mathrm{V}$ and $5 / 48$ cases were given BIRADS score VI). On the other hand, 9/48 cases were classified as benign lesions (2/48 cases were given BIRADS score II, 7/48 cases were given BIRADS score III) and considered $42 / 90$ cases as negative (BIRADS I).

While DBT detected lesions in $73 / 90$ cases $(81.1 \%)$ from which classified $46 / 73$ cases as with malignant lesions (25/73 cases were given BIRADS score IV, 16/73 cases were given BIRADS score $\mathrm{V}, 5 / 73$ cases were given BIRADS score VI), whereas 27/73 cases were considered as with benign lesions (4/73 cases were given BIRADS score II, 23/73 patients were given BIRADS score III) and $17 / 90$ cases as negative (BIRADS score I). This is shown in (Table 2)

With correlation with the final diagnosis 17 cases were true negative and 73 cases were true positive for the presence of breast lesions from which 45 cases were malignant with invasive duct carcinomas detected in 44/ $45(97.7 \%)$ and DCIS associated IDC in 1/45 (2.2\%) with 28 cases were benign breast lesions ( cysts, fibroadenoma, duct ectasia, and intramammary LNs).

By adding DBT to FFDM 52/90 cases were changed their BIRADS scoring as follows: 13 cases were upgraded from BIRADS I to IV, 14 cases were upgraded from BIRADS I to III, 12 cases were upgraded from BIRADS IV to $\mathrm{V}$, and 4 cases were upgraded from BIRADS III to IV.

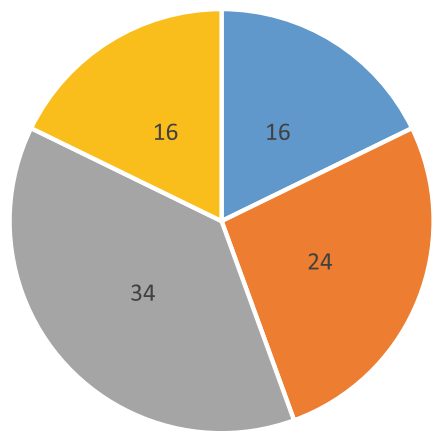

(total 90 cases)

$$
\begin{array}{ll}
\| \text { Almost entirely fatty (ACR a) } & \text { - Scattered fibroglandular density(ACR b) } \\
- \text { Heterogeneously dense (ACR c) } & \quad \text { Extremely dense (ACR d) }
\end{array}
$$

Fig. 1 Breast density wise distribution of study population 


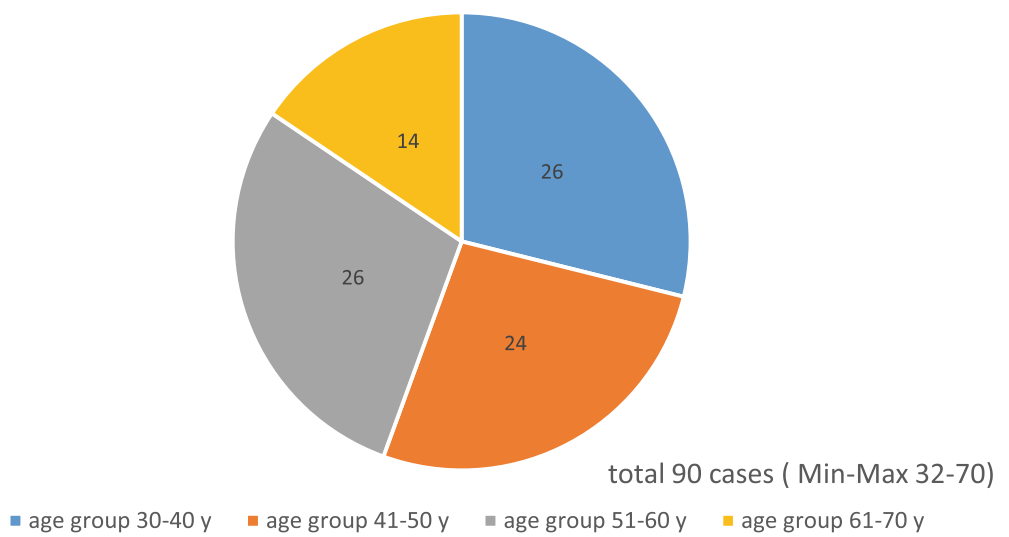

Fig. 2 Age-wise distribution of the study population

Downgrading BIRADS scoring detected in 7 cases from IV to II and III and two cases were downgraded from BIRADS IV to I.

In 38 cases, DBT did not change the BIRADS scoring, but its addition increased the diagnostic confidence and better evaluation of the lesions detected.

After revising the results of FFDM with the final diagnosis by other modalities, histopathology, and/or close follow-up, 29 cases were true positive, 10 cases were false positive, 16 cases were false negative, and 35 cases were true negative. Diagnostic indices of mammography were a sensitivity of $64.44 \%$, a specificity of $77.78 \%$, a positive predictive value of $74.63 \%$, a negative predictive value of $68.63 \%$, and a diagnostic accuracy of $71.11 \%$. While for DBT 45 cases were true positive, 1 case was false positive, no cases were false negative, and 44 cases were true negative. Diagnostic indices were a sensitivity of $100 \%$, a specificity of $97.77 \%$, a positive predictive value of $97.78 \%$, a negative predictive value of $100 \%$, and a diagnostic accuracy of $97.7 \%$. This is shown in Table 3.

In patients with fatty breasts ( ACR a) $16 / 90$ patients, in screening setup, $72 \%$ of cases had no change in BIRADS scoring with the addition of DBT, and $14 \%$ of cases had downgraded and 14\% had upgraded their BIRADS scoring. In diagnostic setup, there was no change in BIRADS scoring with the addition of DBT but increased diagnostic confidence. While in patients with scattered fibro-glandular breast densities (ACR b), in screening setup, $75 \%$ of cases had upgraded BIRADS scoring with the addition of tomosynthesis and $25 \%$ of cases had no change in BIRADS scoring with the addition of DBT. In diagnostic setup, $25 \%$ of cases had downgraded, $12.5 \%$ of cases had upgraded BIRADS scoring with the addition of tomosynthesis, and $62.5 \%$ of cases had no change in BIRADS scoring with the addition of DBT (Fig. 4).

Patients with heterogeneously dense breasts (ACR c), in screening setup, $37.5 \%$ of cases had upgraded BIRADS

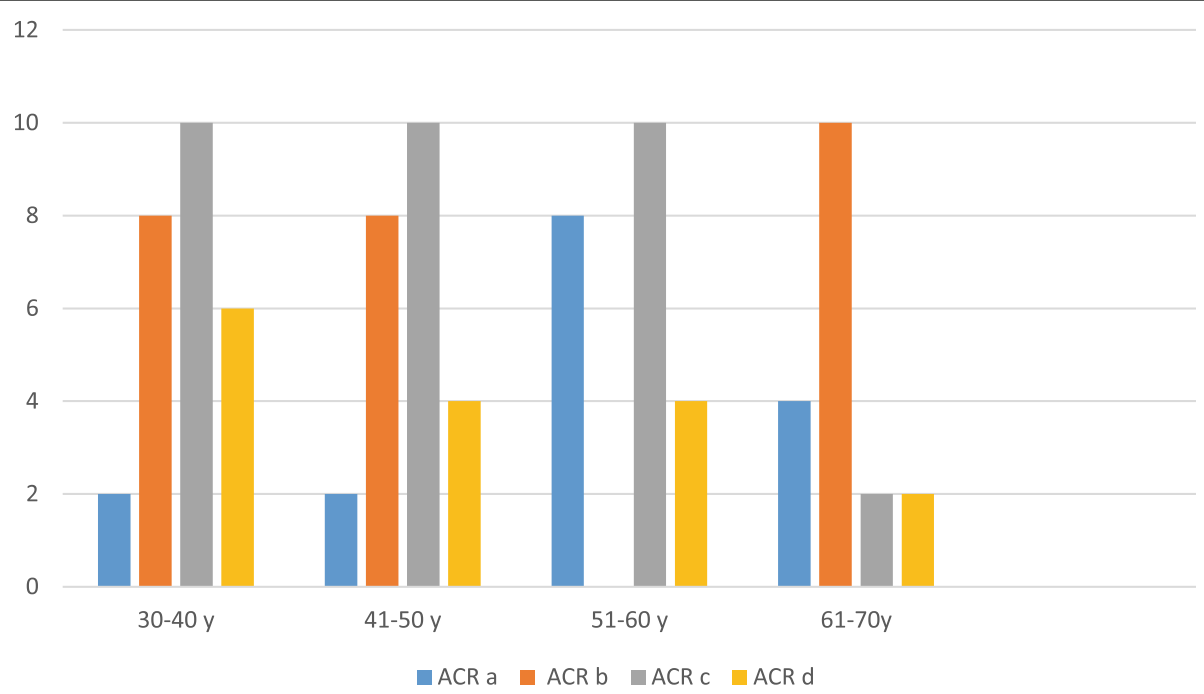

Fig. 3 Distribution of breast densities among different age groups 
Table 2 Comparison of lesion detectability and classification between FFDM and DBT

\begin{tabular}{lllll}
\hline & FFDM & DBT & \\
\hline Positive cases & $48 / 90$ cases & & $73 / 90$ cases \\
Classification of the lesions & Malignant (BIRADS IV, V \& VI) & Benign BIRADS (II \& III) & Malignant (BIRADS IV, V, \& VI) & Benign BIRADS (II \& III) \\
& $39 / 48$ cases & $9 / 48$ cases & $46 / 73$ cases & $27 / 73$ cases \\
\hline
\end{tabular}

scoring, $12.5 \%$ of cases had downgraded BIRADS scoring with the addition of tomosynthesis, and $50 \%$ of cases had no change in BIRADS scoring. In diagnostic setup, $44 \%$ of cases had upgraded BIRADS scoring, $22 \%$ of cases had downgraded BIRADS scoring with the addition of tomosynthesis, and $34 \%$ of cases had no change in BIRADS scoring (Fig. 5).

However, in patients with extremely dense breasts (ACR d), in screening setup, 100\% of cases had upgraded BIRADS scoring with the addition of tomosynthesis, and in diagnostic setup, $71.5 \%$ of cases had upgraded BIRADS scoring, and $28.5 \%$ had no change in BIRADS scoring with the addition of DBT (Fig. 6).

So in non-dense breasts (ACR a and b), 55\% of cases had no change in BIRADS scoring, with $30 \%$ of cases had upgraded BIRADS scoring and 15\% of cases had downgraded BIRADS scoring with the addition of tomosynthesis. Yet, in patients with dense breasts (ACR c and d), $61 \%$ of cases changing their BIRADS scoring with the addition of tomosynthesis (49\% of cases had upgraded and $12 \%$ of cases had downgraded) and $39 \%$ of cases had no change in BIRADS scoring with the addition of DBT (Fig. 7).

Patients in the age group 30-40 years, in diagnostic setup, $73 \%$ cases had changed BIRADS scoring ( $46 \%$ of cases had upgraded and $27 \%$ of cases had downgraded) and $27 \%$ had no change in BIRADS scoring with the addition of DBT. In screening setup, 100\% of cases had no change in BIRADS scoring with the addition of tomosynthesis yet increased diagnostic confidence. Patient of the age group 41-50 years, in diagnostic setup, $60 \%$ of cases had no change in BIRADS scoring, $40 \%$ of cases had upgraded in BIRADS scoring with the addition of tomosynthesis. In screening setup, $57 \%$ of cases had changed BIRADS scoring (43\% of cases had upgraded and $14 \%$ had downgraded) and $43 \%$ of cases had no change in BIRADS scoring with the addition of tomosynthesis.

Patient in the age group 51-60 years, in diagnostic setup, $72 \%$ of cases had no change in BIRADS scoring, yet $28 \%$ of cases had upgraded BIRADS scoring with the addition of tomosynthesis. In screening setup, $50 \%$ of cases had no change in BIRADS scoring, 33\% of cases had upgraded, and $17 \%$ of cases had downgraded BIRADS scoring with the addition of tomosynthesis. Patients with age ranging from 61-70 years came for diagnostic set up had no change in BIRADS scoring, yet, at screening setup, $67 \%$ of cases had no change in BIRADS scoring and 33\% of cases had upgraded BIRADS scoring with the addition of tomosynthesis (Fig. 8).

The diagnostic results of FFDM and DBT for each breast density and age group were evaluated according to sensitivity, specificity, PPV, NPV, and $P$ value (Table 4).

As regards detection of calcification, FFDM detected calcification in 40 cases and classified them as 16 cases

Table 3 Comparison of a diagnostic performance and indices of mammography and tomosynthesis

\begin{tabular}{|c|c|c|c|c|c|c|}
\hline & & Gold standard & & & & $P$ value \\
\hline & & Malignant & & Benign & & \\
\hline & & Count & $\%$ & Count & $\%$ & \\
\hline \multirow[t]{2}{*}{ Mamo (lesions classification) } & Malignant & 29 & 64.4 & 10 & 22.2 & $<0.001$ \\
\hline & Benign & 16 & 35.6 & 35 & 77.8 & \\
\hline \multirow[t]{2}{*}{ Tomo (lesions classification) } & Malignant & 45 & 100.0 & 1 & 0.02 & $<0.001$ \\
\hline & Benign & 0 & 0.0 & 44 & 97.77 & \\
\hline \multirow[t]{2}{*}{ Statistic } & Mammography & & & Tomography & & \\
\hline & Value (\%) & \multicolumn{2}{|l|}{$95 \% \mathrm{Cl}$} & Value (\%) & $95 \% \mathrm{Cl}$ & \\
\hline Sensitivity & 64.44 & \multicolumn{2}{|l|}{48.78 to $78.13 \%$} & 100.00 & \multicolumn{2}{|l|}{92.13 to $100.00 \%$} \\
\hline Specificity & 77.78 & \multicolumn{2}{|l|}{62.91 to $88.80 \%$} & 97.77 & \multicolumn{2}{|l|}{92.13 to $100.00 \%$} \\
\hline Positive Predictive Value & 74.36 & \multicolumn{2}{|l|}{61.69 to $83.93 \%$} & 97.78 & \multicolumn{2}{|l|}{-} \\
\hline Negative Predictive Value & 68.63 & \multicolumn{2}{|l|}{58.89 to $76.96 \%$} & 100.00 & \multicolumn{2}{|l|}{ - } \\
\hline Accuracy & 71.11 & \multicolumn{2}{|l|}{60.60 to $80.18 \%$} & 97.77 & \multicolumn{2}{|l|}{95.98 to $100.00 \%$} \\
\hline
\end{tabular}



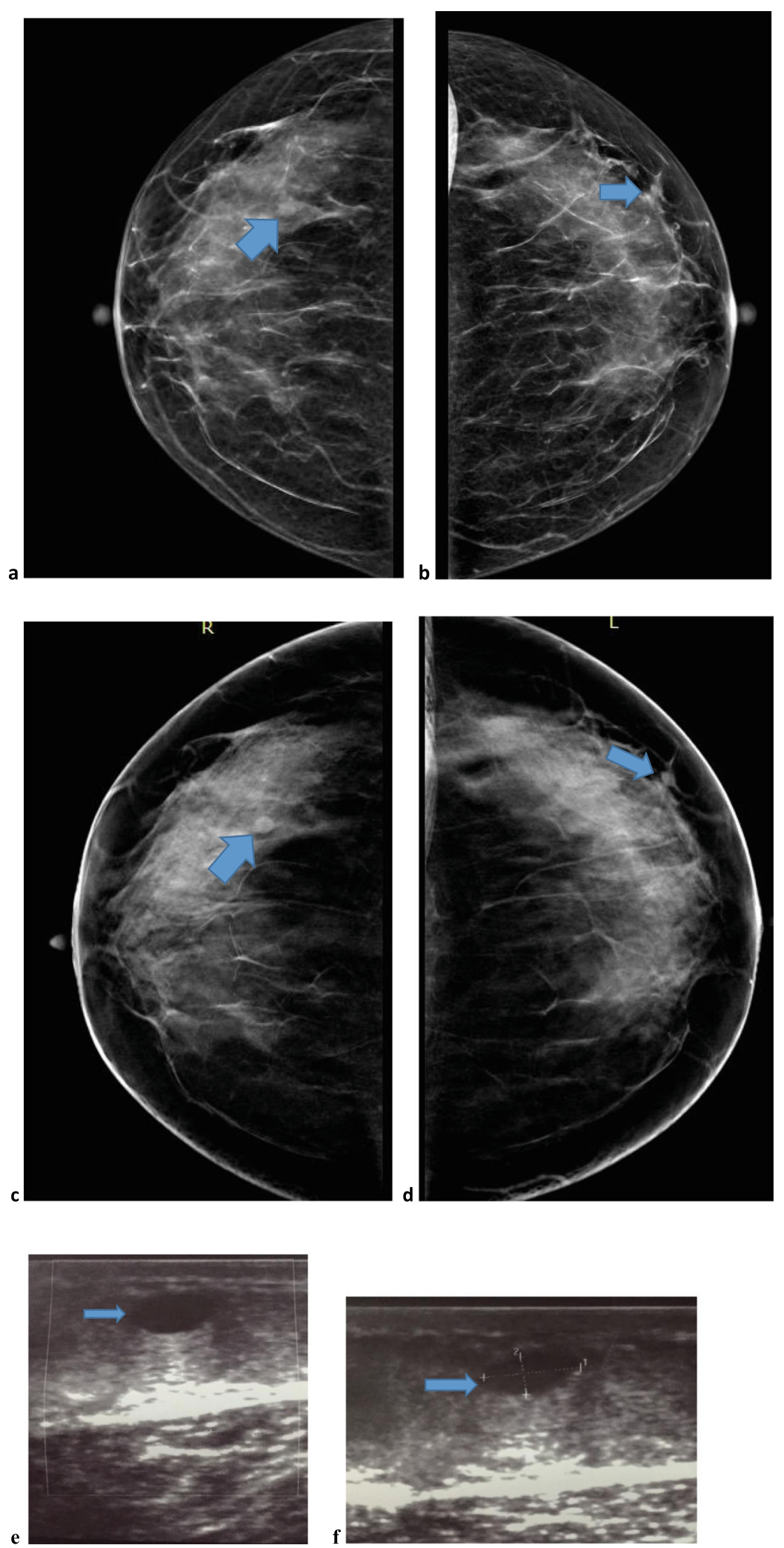

Fig. 4 (See legend on next page.) 
(See figure on previous page.)

Fig. 4 A 38-year-old patient, presented by bilateral breast pain. a, b FFDM in CC views of both breasts: both breasts show scattered fibroglandular parenchyma (ACR b). Right UOQ shows rounded lesion with indistinct border and left UOQ shows oval mass with partly obscured margins (arrows). No suspicious calcifications are seen (BIRADS IV). c, d DBT CC views of both breasts. Both breasts show oval masses with welldefined margins at corresponding sites of mammography (BIRADS III). e, f US examination shows well-defined anechoic cysts at the corresponding site of the lesions detected by tomosynthesis and mammography. FNAC proved bilateral simple cysts

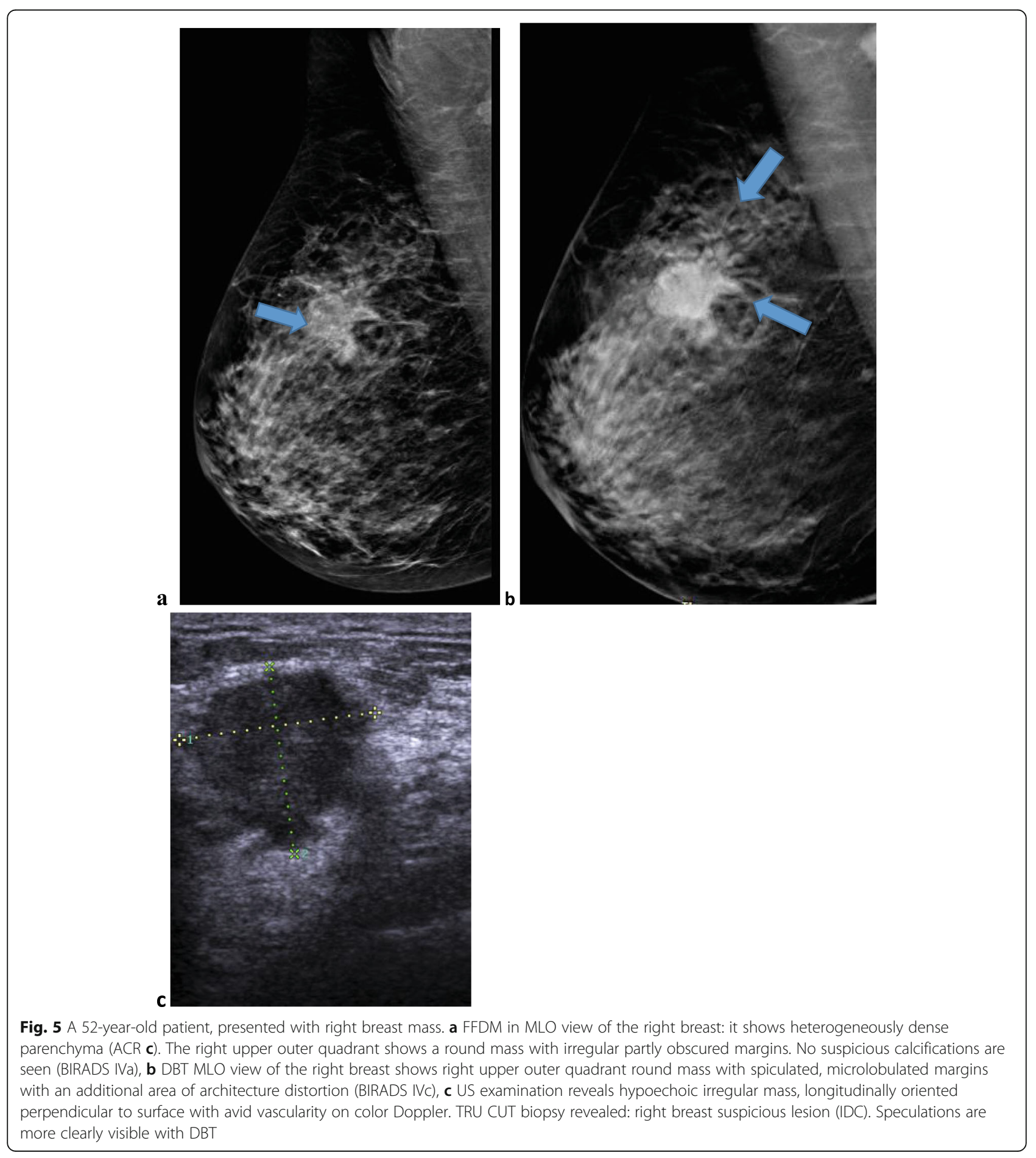




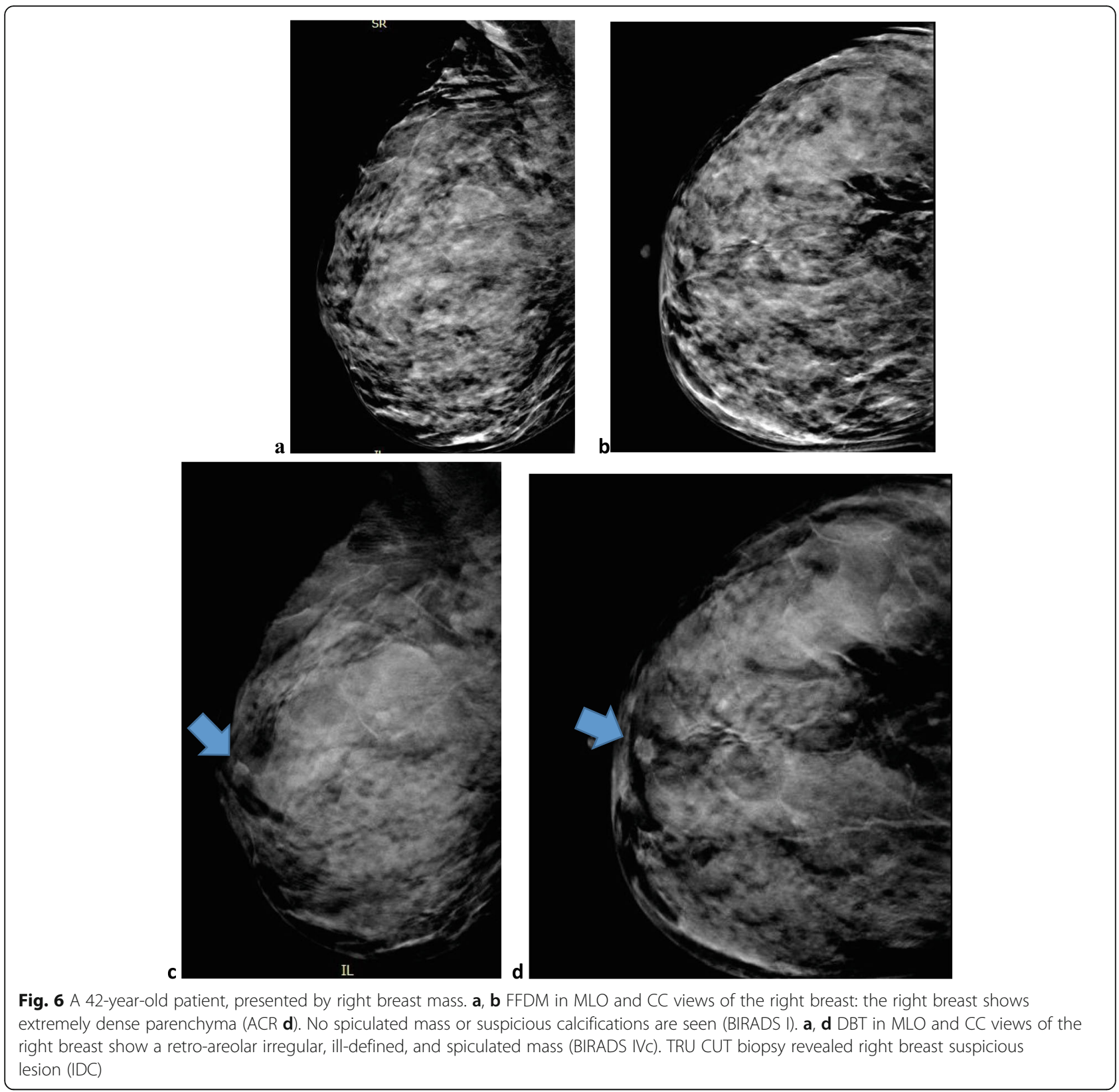

contained suspicious calcification in the form of fine pleomorphic calcification and 24 cases contained benign calcification in the form of popcorn, dystrophic, and vascular calcification. Yet, DBT detected calcification in 33 cases only and classified them as 12 cases contained suspicious micro calcification and 21 cases contained benign-looking calcification.

All detected calcifications were associated with detectable masses and were not the only positive finding in any case. A diagnostic analysis for detection of calcification per se was done and revealed that the sensitivity of DBT was $16 \%$ lower than FFDM in the detection of calcification.

\section{Discussion}

Mammogram has been the gold standard technique and the mainstay for the detection of breast cancer over decades [8]. Women with the dense breasts meet two major problems, as increased breast density decreases the sensitivity and specificity of mammography owing to a decrease in the contrast between tumor and surrounding breast tissue, and superimposed breast tissues may obscure lesions, resulting in a considerable number of false-negative mammograms. Furthermore, the dense breast itself is a risk factor for developing breast cancer [9]. Tomosynthesis has evolved as advanced imaging technique for early diagnosis of breast lesions with a 


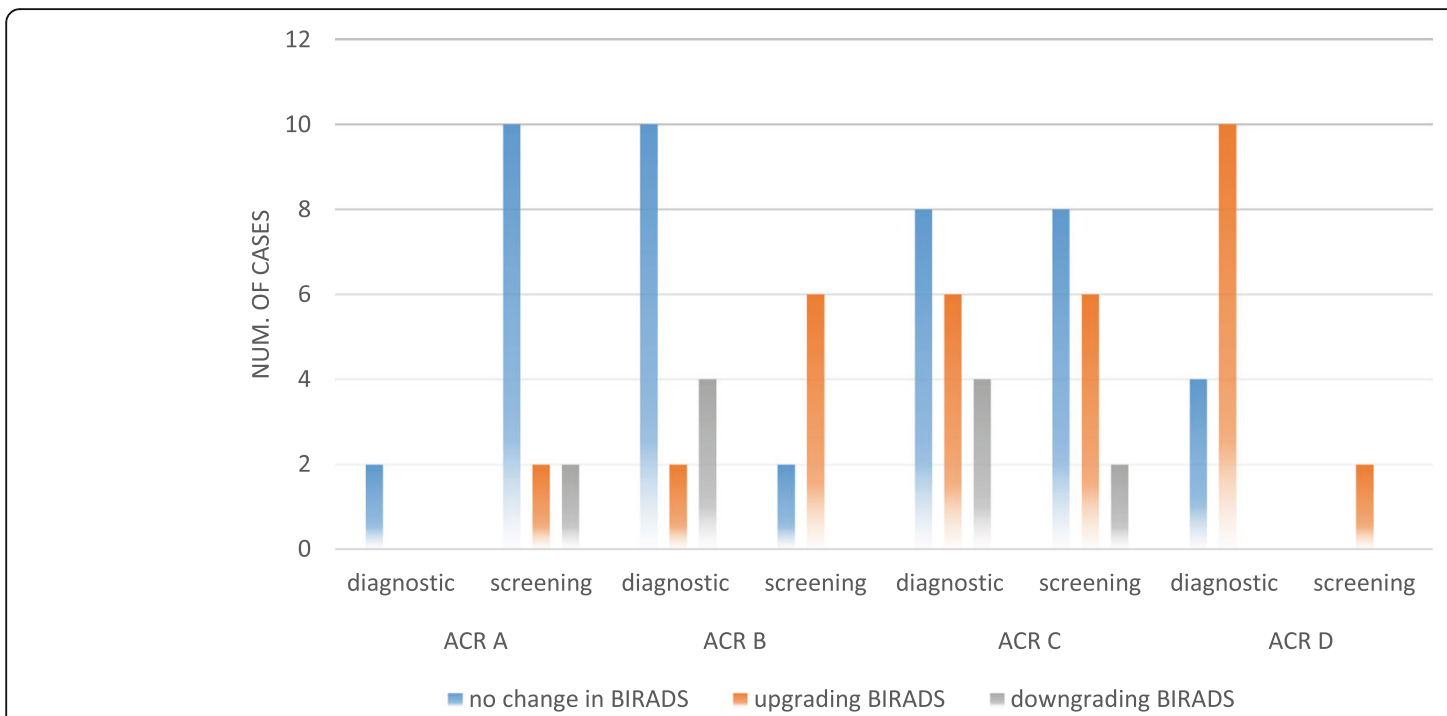

Fig. 7 Effect of addition of DBT to FFDM on BIRADS scoring in different breast densities in diagnostic and screening setup

promising role particularly in dense and treated breasts [8]. Digital breast tomosynthesis provides 3D imaging of the breast, so it reduces the superimposition of breast tissue and improves cancer detection. Previous studies showed that DBT improved the sensitivity, specificity, and accuracy of full-field digital mammography by reducing the recall rate and increasing the cancer detection rate [10].

In the current study, we compared the performance of digital breast tomosynthesis with the performance of FFDM in all breast densities at different age groups. We observed that digital breast tomosynthesis was superior to FFDM in the detection and characterization of different breast lesions in correlation with the final diagnosis.
We also observed improvement in the overall diagnostic capability by adding DBT to FFDM. Sensitivity of lesion detection by DBT was $100 \%$ compared to $64.44 \%$ by FFDM, specificity $97.77 \%$ by DBT compared to $77.78 \%$ by FFDM, positive predictive value $97.78 \%$ by DBT compared to $74.63 \%$ by FFDM, negative predictive value $100 \%$ by DBT compared to $68.63 \%$ by FFDM, and a diagnostic accuracy $97.7 \%$ by DBT compared to $71.11 \%$ by FFDM.

Our results were comparable to prior studies done by Conant et al. [11], Skaane et al. [12], and Divya et al. [13] who reported increased sensitivity, specificity, and increased cancer detection when DBT was used. Skaane et al. found increase sensitivity from 54.1 to $70.5 \%$ and

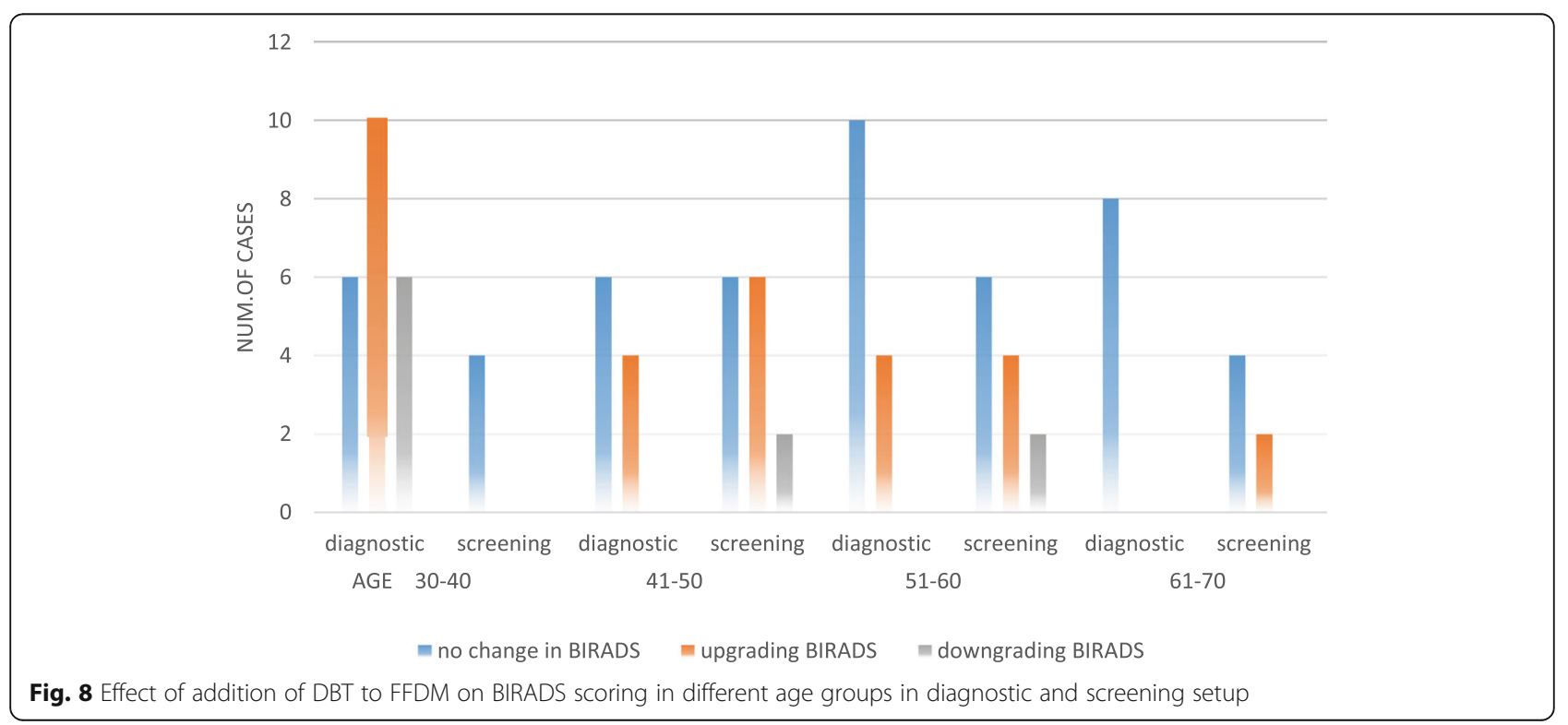


Table 4 Comparison of diagnostic indices of mammography and tomosynthesis in different breast densities and age groups (significant $P$ value $<0.05$ )

\begin{tabular}{|c|c|c|c|c|c|c|c|c|c|c|}
\hline & & \multicolumn{2}{|l|}{ Sensitivity } & \multicolumn{2}{|l|}{ Specificity } & \multicolumn{2}{|l|}{ PPV } & \multicolumn{2}{|l|}{ NPV } & \multirow[t]{2}{*}{$P$ value } \\
\hline & & FFDM (\%) & DBT (\%) & FFDM (\%) & DBT (\%) & FFDM (\%) & DBT (\%) & FFDM (\%) & DBT (\%) & \\
\hline$\overline{\mathrm{ALL}}$ & & 64.4 & 100.0 & 77.8 & 97.77 & 74.4 & 97.77 & 68.6 & 100.0 & $<0.001$ \\
\hline \multirow[t]{4}{*}{ ACR } & $\mathrm{a}$ & 86.7 & 100.0 & 83.3 & 100.0 & 66.7 & 100.0 & 83.3 & 100.0 & 0.455 \\
\hline & b & 60.0 & 100.0 & 50.0 & 100.0 & 60.0 & 100.0 & 50.0 & 100.0 & 0.087 \\
\hline & c & 76.5 & 100.0 & 70.0 & 97.22 & 76.5 & 97.22 & 80.0 & 100.0 & 0.013 \\
\hline & d & 50.0 & 100.0 & 100.0 & 100.0 & 100.0 & 100.0 & 45.5 & 100.0 & 0.014 \\
\hline \multirow[t]{4}{*}{ age } & $30-40$ & 75.0 & 100.0 & 68.4 & 96.3 & 50.0 & 96.33 & 86.7 & 100.0 & 0.046 \\
\hline & $41-50$ & 33.3 & 100.0 & 84.6 & 100.0 & 66.7 & 100.0 & 57.9 & 100.0 & 0.001 \\
\hline & $51-60$ & 68.4 & 100.0 & 77.78 & 100.0 & 86.7 & 100.0 & 53.8 & 100.0 & 0.020 \\
\hline & $61-70$ & 100.0 & 100.0 & 100.0 & 100.0 & 100.0 & 100.0 & 100.0 & 100.0 & --- \\
\hline
\end{tabular}

mild increase in specificity from 94 to $95 \%$ by using DBT compared to digital mammography [12]. While Divya et al. showed that sensitivity increased from 83.6 to $100 \%$, also specificity increased from 38.7 to $87.77 \%$ and positive predictive value raised from of 56 to $81.67 \%$ after adding DBT to FFDM [13].

In this current study, we observed that 52/90 (57.7\%) of cases demonstrated a discrepancy between FFDM and DBT in BIRADS scoring. By adding DBT to FFDM, 13 cases were upgraded from BIRADS I to IV by discovering spiculated lesions and/or architecture distortion in DBT proved by US examination and histopathology were not seen in mammography as patients were with high-density breasts. Another 14 cases were upgraded from BIRADS I to III by discovering well-defined lesions on tomosynthesis proved by the US to be cysts, fibroadenomas, and/or intramammary LNs which could not be seen at mammography by the effect of overlapping breast tissue in the dense breast. Four cases were upgraded from BIRADS III to IV detected at mammography as well defined masses, yet discovering speculation, indistinct border, and/or architecture distortion in tomosynthesis as it reduces the superimposition of the breast tissue and proved by the US examination and histopathology to be IDC. Also 12 cases were upgraded from BIRADS IV to V by finding frank speculation and dense rounded lymphadenopathies proved by US and histopathology to be IDC.

In seven cases, BIRADS scoring were downgraded from IV to II and III by observing a well-defined border by tomosynthesis, which minimizes the effect of tissue overlap and allows better visualization of the masses reported as of indistinct border by mammography. US examination proved them well-defined cysts and/or fibroadenomas. Two cases were downgraded from BIRADS IV to I by proving no evidence of the architecture distortion reported by mammography in DBT and proved by the US examination.
In thirty-eight cases, DBT did not change the BIRADS scoring, but its addition increased the conspicuity of the lesions detected and diagnostic confidence.

Our results were comparable to a study performed by Divya et al. [13], which conducted a comparison between DBT and FFDM according to the BIRADS scoring. With the addition of tomosynthesis to FFDM 53\% of the cases changing their BIRADS scoring, BIRADS was upgraded in $14 \%$ and downgraded in $31 \%$ of cases and DBT did not change the BIRADS scoring in $47 \%$ of cases but its addition increased the diagnostic confidence.

In our study, we noticed the change in BIRADS scoring was detected mainly in patients with dense breasts. As patients with high breast densities (ACR $\mathrm{c}$ and d), $61 \%$ of cases had changed their BIRADS scoring (49\% of cases had upgraded and 12\% of cases had downgraded) with the addition of tomosynthesis and 39\% of cases had no change in BIRADS scoring ( $P$ value 0.04 ). Yet, in patients with fatty and fibro-glandular breast densities (ACR a and b), 30\% of cases had upgraded and 15\% of cases had downgraded BIRADS scoring with the addition of tomosynthesis with $55 \%$ of cases had no change in BIRADS scoring ( $P$ value 0.3 ).

The results of our study are comparable with the study of Østerås et al. [14]. He said that adding DBT to FFDM yields more speculated masses or architectural distortions in women of all density categories with $P$ value < .001 in women with scattered fibro-glandular and heterogeneously dense breasts and $P$ value $>.05$ in women with almost entirely fatty and extremely dense breasts.

As reported in several earlier studies, evidence suggests that the sensitivity and specificity of mammography decreased in the breasts with a higher density. In our study, the sensitivity of FFDM in dense breasts (ACR c and d) is $76.7 \%$ compared to $84.5 \%$ in non-dense breasts ACR ( $a$ and b), yet DBT had a sensitivity of $100 \%$ in both dense and non-dense breasts. The 
specificity of FFDM in the dense breasts was 70.5\% compared to $83.6 \%$ in non-dense breasts, yet DBT had a specificity of $97.7 \%$ in dense breasts and $100 \%$ in non-dense breasts.

Chiu et al. [15] reported in their study about the effect of breast density on breast cancer incidence and screening. The sensitivity of mammography was $62.8 \%$ in dense breasts as compared to $82 \%$ in non-dense breasts and a drop in specificity was reported to be $89.6 \%$ in dense breasts as compared to $96.5 \%$ in non-dense breasts.

Phi et al. [10] and Asbeutah et al. [16] performed a meta-analysis on tomosynthesis in dense breasts for screening and diagnosis. They concluded that DBT increased sensitivity and the cancer detection rate in screening and diagnosis in comparison to mammography alone.

In our study, we noticed that DBT detected more breast masses than did FFDM. DBT detected new lesions in 22 cases especially in patients with dense breasts (ACR $\mathrm{c}$ and $\mathrm{d}$ ) and allows better visualization and evaluation of lesions margins.

Our results supported by the literature of Conant et al. [11] in their study about digital breast tomosynthesis vs digital mammography in cancer detection and recall rates in different ages and breast densities. He concluded that screening with DBT simultaneously improved breast cancer detection while reducing falsepositive recalls which were significant across all age groups and breast densities categories. Invasive cancers detected by DBT were more likely smaller and nodenegative compared with cancers detected by digital mammography, particularly in women aged 40 to 49 years.

Rafferty et al. [17] performed a study that included digital mammography alone and digital mammography plus tomosynthesis for breast cancer screening in the dense and non-dense breasts. They concluded that adding tomosynthesis to digital mammography leads to an increase in cancer detection rate and reduction in recall rate more obvious in women with heterogeneously dense breasts than in those with non-dense breasts or extremely dense breasts.

In our study, we discovered that in patients of the age group 30-40 years (complaining of breast masses or for screening for breast cancer due to 1st-degree relative positive for breast cancer), $61.5 \%$ had changed their BIRADS scoring with the addition of DBT ( $P$ value 0.02 ). In patients of age group 4150 years, $50 \%$ of cases changed BIRADS scoring with the addition of tomosynthesis ( $P$ value 0.07 ). Yet, in patients of age group 51-60 years, $61.5 \%$ of cases had no change in BIRADS scoring ( $P$ value 0.08 ), and patients in the age group of $61-70$ years, $85 \%$ of cases had no change in BIRADS scoring with the addition of DBT but increased diagnostic confidence ( $P$ value 0.8 ).

The sensitivity of FFDM in pts. $<50$ years is $54.15 \%$ compared to $84.2 \%$ in pts. $>50$ years (as most patients below 50 years were female had dense breast or on hormonal contraceptive method) with sensitivity of DBT $100 \%$ in both. The specificity of FFDM in pts. $<50$ years is $76.5 \%$ compared to $88.6 \%$ in pts. $>50$ years with a specificity of DBT in pts. $<50$ years and $96.31 \%$ compared to $100 \%$ in pts. $>50$ years.

The study of Østerås et al. [14] was comparable to our results which concluded that the results of DBT were higher for all age strata compared to FFDM. Improvement in the true-positive rate and decrease in falsepositive interpretations with $P=.008$ for ages less than 50 years, $P=.02$ for ages $51-59$ years, and $P=.001$ for ages 60-64 years.

We noticed in our study that there is a $33 \%$ reduction in recall rate after adding tomosynthesis compared to FFDM alone. We observed that across all breast densities and patient ages with statistical significance in all subgroups except in patients with predominantly fatty breast density and patients older than 60 years. Patients receiving the greatest benefit were women with dense breasts (ACR $\mathrm{c}$ and $\mathrm{d}$ ) and those younger than 50 years.

The aforementioned results were in keeping with Haas et al. [18]who concluded that recall rates were much lower in patients who underwent tomosynthesis plus conventional mammography than in those who underwent conventional mammography alone with a $30 \%$ reduction in recall rate overall.

Divya et al. [13] concluded that the most significant decrease in recall rates was due to a reduction in falsepositive patients. Combination of the two modalities leads to reduction in BIRADS in 31\% of patients with a significant decrease in recall rates seen in ACR categories b, c, d, and age groups $<40$ years due to improved sensitivity, specificity, positive predictive value, and reduced false-positive value.

The study done by Lowry et al. [19] has a comparative effectiveness study between the performance of tomosynthesis vs digital mammography in community practice by patient age, screening round, and breast density. They concluded that both recall and cancer detection rates improved using digital breast tomosynthesis vs digital mammography for women aged 40 to 79 years with heterogeneously dense breasts and for women aged 50 to 79 years with scattered fibro-glandular density; by contrast, the performance was similar in women with extremely dense breasts.

In our study, we found that FFDM is superior to DBT in detection calcification with a sensitivity of DBT $16 \%$ lower than FFDM. However, the results of the synthetic 2D image are matching with that of FFDM. 
Spangler et al. [20] in their study said that FFDM appears slightly more sensitive than digital breast tomosynthesis for the detection of calcification. However, diagnostic performance using BI-RADS was not much different.

According to the results of our study, we recommend tomosynthesis as the 1st step diagnostic and screening tool instead of mammography in young females below 40 years and in females with proposed dense breast as on hormonal therapy or hormonal contraception, which may have a favorable risk to benefit ratio.

Tomosynthesis proved to be of higher sensitivity and specificity than FFDM in all breast densities, especially dense breasts. It has the advantages of three-dimensional data, which helps in eliminating the overlap of tissues seen in two-dimensional images of mammography with better resolution of the internal breast contents leading to better diagnostic capabilities. This in turn would reduce false-positive results, unnecessary recalls, and the need for additional time-consuming imaging such as special mammographic views, thereby increasing the efficacy of DBT by reducing the additional radiation dose, time, and money.

\section{Conclusion}

DBT is a promising imaging modality offering better detection and characterization of different breast abnormalities, especially in young females, and those with dense breasts with an increase of sensitivity and specificity than FFDM. This leads to a reduction in the recalled cases, negative biopsies, and assessing the efficacy of therapy as it enables improving detection of breast cancer and different breast lesions not visualized by conventional mammography.

\section{Abbreviations}

FFDM: Full-field digital mammography; DBT: Digital breast tomosynthesis: DM: Digital mammography; BIRADS: Breast imaging reporting and data system; CC: Craniocaudal; MLO: Mediolateral oblique; 3D: Three-dimensional; SM: Synthetic mammography; ACR: American College of Radiology; RR: Relative risk; PPV: Positive predictive value; NPV: Negative predictive value; LN: Lymph node

\section{Acknowledgements}

The author thanks all the study participants for their patience and support.

\section{Authors' contributions}

RMN collected and analyzed the data, wrote the manuscript, prepared the FFDM and DBT cases, performed the required measurements and statistical analysis, and prepared the figures and tables. MEA suggested the research idea, shared in the data collection and analysis, and reviewed the literature, statistical analysis, and manuscript editing. MAN reviewed the manuscript and statistical analysis. RAM reviewed the manuscript and statistical analysis. The authors read and approved the final manuscript.

\section{Funding}

This study had no funding from any resource.

\section{Availability of data and materials}

The datasets used and analyzed during the current study are available from the corresponding author on reasonable request.

\section{Ethics approval and consent to participate}

Participants included in this research gave written informed consent to publish the data contained within this study.

\section{Competing interests}

The authors declare that they have no competing interests.

Received: 20 November 2020 Accepted: 18 January 2021

Published online: 02 February 2021

References

1. Thigpen D, Kappler A, Brem R (2018) The role of ultrasound in screening dense breasts-a review of the literature and practical solutions for implementation. Diagnostics 8(1):20

2. Jae-Hun K, Eun SK, Yaeji L et al (2017) Breast cancer heterogeneity MR imaging texture analysis and survival outcomes. Radiology 282(3):665-675

3. Tabar L, Vitak B, Chen THH et al (2011) Swedish two-county trial: impact of mammographic screening on breast cancer mortality during 3 decades. Radiology 260:658-663. https://doi.org/10.1148/radiol.11110469

4. Lewin JM, Niklason L (2007) Advanced applications of digital mammography: tomosynthesis and contrast-enhanced digital mammography. Semin Roentgenol 42:243-252

5. Emaus MJ, Bakker MF, Peeters PH et al (2015) MR Imaging as an additional screening modality for the detection of breast cancer in women aged 5075 years with extremely dense breasts: the DENSE trial study design. Radiology 0:141827.4- 24(1):84-88

6. Garcia EM, Crowley J, Hagan C et al (2016) Evolution of imaging in breast cancer. Clin Obstet Gynaecol 59:322-335

7. Ren B, Ruth C, Wu T et al (2010) A new generation FFDM/tomosynthesis fusion system with selenium detector. Proc SPIE 7622:B1-B10

8. D'Orsi CJSE, Mendelson EB, Morris EA (2013) ACR BI-RADS ${ }^{\circledR}$ atlas, breastimaging reporting and data system. American College of Radiology, Reston, VA

9. Nazari SS, Mukherjee P (2018) An overview of mammographic density and its association with breast cancer. Breast cancer 25(3):259-267

10. Phi XA, Tagliafico A, Houssami N et al (2018) Digital breast tomosynthesis for breast cancer screening and diagnosis in women with dense breasts - a systematic review and meta-analysis. BMC Cancer 18(1):380

11. Conant, E. F., Barlow, W. E., Herschorn, S. D et al (2019). Association of digital breast tomosynthesis vs digital mammography with cancer detection and recall rates by age and breast density. JAMA Oncol doi:https://doi.org/10. 1001/jamaoncol.2018.7078

12. Skaane $p$, Bandos AL, Niklason LT et al (2019) Digital mammography versus digital mammography plus tomosynthesis in breast cancer screening: the Oslo tomosynthesis screening trial. Radiology 291(1):23-30

13. Singla D, Chaturvedi AK, Aggarwal A et al (2018) Comparing the diagnostic efficacy of full field digital mammography with digital breast tomosynthesis using BIRADS score in a tertiary cancer care hospital. Indian J Radiol Imaging 28(1):115-122. https://doi.org/10.4103/ijri.JJRI_107_17

14. Østerås BH, Martinsen ACT, Gullien R et al (2019) Digital mammography versus breast tomosynthesis: impact of breast density on diagnostic performance in population-based screening. Radiology:190425. https://doi. org/10.1148/radiol.2019190425

15. Chiu SY, Duffy S, Yen AM et al (2010) Effect of baseline breast density on breast cancer incidence, stage, mortality, and screening parameters: 25-year follow-up of a Swedish mammographic screening. Cancer Epidemiol Biomark Prev 19(5):1219-1228

16. Asbeutah A, Karmani N, Asbeutah A et al (2018) Comparison of digital breast tomosynthesis (DBT) and digital mammography (DM) for detection of breast cancer in women in Kuwait. Med PrincPract 28(1):10-15

17. Rafferty EA, Durand MA, Conant EF et al (2016) (2016) Breast cancer screening using tomosynthesis and digital mammography in dense and non-dense breasts. JAMA 315:1784-1786

18. Haas BM, Kalra V, Geisel J et al (2013) Comparison of tomosynthesis plus digital mammography and digital mammography alone for breast cancer screening. Radiology 269(3):694-700. https://doi.org/10.1148/radiol.13130307 
19. Lowry KP, Coley RY, Miglioretti DL et al (2020) Screening Performance of digital breast tomosynthesis vs digital mammography in community practice by patient age, screening round, and breast density. JAMA Netw Open 3(7):e2011792. https://doi.org/10.1001/jamanetworkopen.2020.11792

20. Spangler ML, Zuley ML, Sumkin JH et al (2011) Detection and classification of calcifications on digital breast tomosynthesis and 2D digital

mammography: a comparison. Am J Roentgenol 196(2):320-324. https:/doi. org/10.2214/ajr.10.4656

\section{Publisher's Note}

Springer Nature remains neutral with regard to jurisdictional claims in published maps and institutional affiliations.

Submit your manuscript to a SpringerOpen ${ }^{\circ}$ journal and benefit from:

- Convenient online submission

- Rigorous peer review

- Open access: articles freely available online

- High visibility within the field

- Retaining the copyright to your article

Submit your next manuscript at $\boldsymbol{\sim}$ springeropen.com 